\title{
Education level and COVID-19 vaccination willingness in adolescents
}

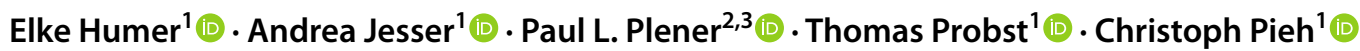

Received: 3 September 2021 / Accepted: 16 September 2021 / Published online: 22 September 2021

(c) Springer-Verlag GmbH Germany, part of Springer Nature 2021

\begin{abstract}
Vaccination is essential to control the COVID-19 pandemic. High vaccination willingness is a key for successful vaccination programs. This study assessed attitudes toward vaccination in Austrian adolescents and determined whether there are differences in vaccination readiness regarding education status, gender and migration background. Two cross-sectional online surveys were conducted from March to July 2021 in apprentices and high school students. Willingness to receive COVID-19 vaccination was rated on a 5-point scale. In total, $n=2006$ ( $n=1442$ apprentices and $n=564$ high school students) completed the survey. Willingness to receive COVID-19 vaccination was higher in students compared to apprentices $(p<0.001)$. Furthermore, migration background $(p=0.023)$ and female gender $(p=0.001)$ were associated with lower vaccination willingness. In conclusion, more efforts are required to improve confidence and willingness to vaccinate adolescents with lower educational levels, those with migrant backgrounds and females.
\end{abstract}

\section{Implications and contribution}

This study implicates that more efforts are required to improve willingness to vaccinate adolescents with lower educational achievements, those with migrant backgrounds and females. Public health campaigns are needed to ensure COVID-19 vaccination efforts also reach those who are currently uncertain or unwilling to get vaccinated.

\section{Introduction}

Recent publications reveal that the COVID-19 pandemic and public health responses to the spread of SARS-CoV-2 (e.g., social distancing, homeschooling, quarantine, isolation) are causing extraordinary psychological distress among adolescents worldwide $[1,2]$. As vaccination is the most effective method to mitigate the disease [3], vaccine willingness is a key for successful vaccination programs. This study aimed

Christoph Pieh

christoph.pieh@donau-uni.ac.at

1 Department for Psychotherapy and Biopsychosocial Health, Danube University Krems, Krems, Austria

2 Department of Child and Adolescent Psychiatry, Medical University of Vienna, Vienna, Austria

3 Department of Child and Adolescent Psychiatry and Psychotherapy, University of Ulm, Ulm, Germany to assess attitudes toward vaccination in a national sample of Austrian adolescents and determine whether there are differences in vaccination readiness regarding education status, gender and migration background.

\section{Methods}

Two cross-sectional surveys were conducted online using Research Electronic Data Capture (REDCap) from March 29, 2021, to May 18, 2021, in apprentices and from June 19, 2021, to July 2, 2021, in high school students.

Demographic data (gender, age, migration background) were collected. Willingness to receive COVID-19 vaccination was measured with a single-item question: "If there was the possibility to get vaccinated against the coronavirus, would you do it?" rated on a 5-point scale from $1=$ "no way" to $5=$ "definitely".

All data were analyzed using the IBM SPSS Statistics software version 26 . Independent $t$ tests and chi-squared tests were computed to analyze differences in sample characteristics (age, gender, migration background). Univariate ANOVA was computed to analyze potential differences in vaccination readiness concerning group (apprentices vs. students), gender (male vs. female) and migration background (yes vs. no). The vaccination willingness was the dependent variable, while group, gender, migration background, as well as their interaction, were included as independent variables. 
Age was included as a covariate in the statistical model. The significance level was set at 0.05 (two-tailed).

\section{Results}

In total, $n=2006$ [ $n=1442$ apprentices $(53.5 \%$ females; $29.1 \%$ migration background, age: $M=18.19$, $\mathrm{SD}=2.30$ years) and $n=564$ high school students (79.6\% females; $12.6 \%$ migration background, age: $\mathrm{M}=16.34, \mathrm{SD}=1.33$ years $)]$ completed the survey. Gender $\left(\chi^{2}(2)=122.109, p<0.001\right)$, migration background $\left(\chi^{2}(1)=59.982, p<0.001\right)$, as well as age $[\mathrm{T}(1$; $1720.733)=22.410 ; p<0.001]$ were not distributed equally between those groups.

Willingness to receive COVID-19 vaccination was higher in students $(M=4.00, \mathrm{SD}=1.31)$ compared to apprentices $(M=3.16, \mathrm{SD}=1.50 ;(F(1)=68.023, p<0.001)$. In addition, $53 \%$ of students and $28 \%$ of apprentices reported a definite willingness to get vaccinated; $7 \%$ of students and $22 \%$ of apprentices reported definite unwillingness to get vaccinated. Furthermore, migration background showed an effect on vaccination readiness $(\mathrm{F}(1)=5.158, p=0.023)$, with participants with migration background reporting a lower willingness to get vaccinated $(M=3.00, \mathrm{SD}=1.48)$ than those without migration background $(M=3.52, \mathrm{SD}=1.48)$. Higher vaccination willingness was observed in male $(M=3.50$, $\mathrm{SD}=1.49)$ compared to female $(M=3.33, \mathrm{SD}=1.50)$ adolescents $(F(1)=10.703, p=0.001)$.

No two-way interactions between group and gender, group and migration background, gender and migration background, as well as no three-way interaction between group, gender and migration background were observed (all $p$ values $\geq 0.149$ ).

\section{Discussion}

Although the COVID-19 course is significantly milder in adolescents than in adults, it is important to include adolescents as targets for vaccination to protect other, more vulnerable populations and help mitigate the COVID-19 pandemic [4]. Vaccination readiness and vaccine hesitancy are key factors for vaccination coverage. However, there is scarce research addressing adolescent vaccination readiness. Brandt et al. [5] assessed adolescents' attitudes towards COVID-19 vaccination in the US in October 2020. They concluded that although $76 \%$ of their 911 14- to 24-year-old respondents reported a willingness to get vaccinated, many had concerns about side effects, efficacy, and safety and would wait until they believed it was safe and recommended by experts. A study conducted in Canada in 664 16- to 21 -year-olds conducted between
November and December 2020 observed a vaccination willingness of $65.4 \%$, with no difference by gender, age, but other sociodemographic characteristics, such as household income and parent education [6].

One major finding of our study was, that education status affected adolescents' willingness to receive vaccination, with high school students reporting higher willingness than apprentices of the same age. This is in line with results from Afifi et al. [6], as well as a review of 126 surveys by Lin et al. [7], showing lower vaccine acceptance among less-educated adults.

In addition to education level, our study revealed lower vaccination willingness in migrants. This is confirmed by the study conducted in the US, which also found that migration background was a predictor for vaccination unwillingness in adolescents [5].

Lastly, we found gender to influence vaccine acceptance, with male adolescents showing a higher willingness to vaccinate. Previous studies on gender and COVID-19 vaccine acceptance are inconclusive [7]. As summarized by Bono et al. [8], some studies support lower vaccination willingness by females, likely due to their higher fear of side effects.

Overall, the results of our study are reflected in observations from the research on adults. A systematic review and meta-analysis by Robinson et al. [9] revealed that being female, younger, of lower income or education level and having a migrant background impacted negatively on the intention to vaccinate.

While interpreting the results, the following limitations should be considered. First, the sample might not be representative of all students and apprentices, i.e., concerning gender, migration background and region. Second, the student sample was rather small. Third, vaccination willingness was assessed with a single item. Fourth, the question about the willingness to get vaccinated against COVID-19 was not further differentiated by COVID-19 vaccines.

As a conclusion from our findings, we suggest that adolescents should be empowered in their health care autonomy [10] by providing youth-friendly materials that ensure adolescents feel informed and able to make important health decisions for themselves. More efforts are required to improve confidence and willingness to vaccinate adolescents with lower educational achievements, those with migrant backgrounds and females. Public health campaigns are needed to ensure vaccination efforts also reach those who are currently uncertain or unwilling to get vaccinated.

Author contributions Conceptualization: Pieh, Plener, Humer, Probst. Data curation: Humer. Statistical analysis: Humer. Investigation: Pieh, Plener, Humer, Probst. Methodology: Pieh, Humer, Plener. Project administration: Pieh, Humer. Writing — original draft: Humer, Jesser. Writing—review and editing: Pieh, Probst, Plener.

Funding None. 
Data availability The raw data supporting the conclusion of this article will be made available by the authors upon reasonable request.

\section{Declarations}

\section{Conflict of interest None.}

Ethics approval The principles outlined in the Declaration of Helsinki were followed and the ethics committee of the Danube-University Krems as well as the data protection officer of the Danube-University Krems approved the study (EK GZ 41/2018-2021).

Consent to participate All participating adolescents had to agree to the data protection declaration to start the survey (electronic informed consent).

Consent for publication Participants gave informed consent for publication.

\section{References}

1. Hoekstra PJ (2020) Suicidality in children and adolescents: lessons to be learned from the COVID-19 crisis. Eur Child Adolesc Psychiatry 29:737-738. https://doi.org/10.1007/s00787-020-01570-z

2. Pieh C, Dale R, Plener PL et al (2021) Stress levels in high-school students after a semester of home-schooling. Eur Child Adolesc Psychiatry. https://doi.org/10.1007/s00787-021-01826-2

3. Mahase E (2020) Covid-19: Expedite vaccination or deaths will surge, researchers warn. BMJ m4958. https://doi.org/10.1136/bmj. $\mathrm{m} 4958$
4. Zimet GD, Silverman RD, Fortenberry JD (2021) Coronavirus disease 2019 and vaccination of children and adolescents: prospects and challenges. J Pediatr 231:254-258. https://doi.org/10. 1016/j.jpeds.2020.11.002

5. Brandt EJ, Rosenberg J, Waselewski ME et al (2021) National study of youth opinions on vaccination for COVID-19 in the US. J Adolesc Health 68:869-872. https://doi.org/10.1016/j.jadohealth. 2021.02.013

6. Afifi TO, Salmon S, Taillieu T et al (2021) Older adolescents and young adults willingness to receive the COVID-19 vaccine: implications for informing public health strategies. Vaccine 39:3473-3479. https://doi.org/10.1016/j.vaccine.2021.05.026

7. Lin C, Tu P, Beitsch LM (2020) Confidence and receptivity for COVID-19 vaccines: a rapid systematic review. Vaccines 9:16. https://doi.org/10.3390/vaccines 9010016

8. Bono SA, de Moura F, Villela E, Siau CS et al (2021) Factors affecting COVID-19 vaccine acceptance: an international survey among low- and middle-income countries. Vaccines 9:515. https:// doi.org/10.3390/vaccines 9050515

9. Robinson E, Jones A, Lesser I, Daly M (2021) International estimates of intended uptake and refusal of COVID-19 vaccines: a rapid systematic review and meta-analysis of large nationally representative samples. Vaccine 39:2024-2034. https://doi.org/10. 1016/j.vaccine.2021.02.005

10. Schuiteman S, Chua K-P, Plegue MA et al (2020) Self-Management of health care among youth: implications for policies on transitions of care. J Adolesc Health 66:616-622. https://doi.org/ 10.1016/j.jadohealth.2020.01.009 